\title{
Transurethral Microwave Thermotherapy for Treatment of Benign Prostatic Hyperplasia: A Preliminary Assessment of the Prostalund Feedback Treatment Machine
}

\author{
Elijah O. Kehinde ${ }^{a, c}$ Fawzi Abul ${ }^{b, c}$ \\ Department of Surgery, Division of Urology, ${ }^{a}$ Mubarak Al-Kabeer Teaching Hospital and ${ }^{b}$ Amiri Hospital, and \\ ${ }^{c}$ Faculty of Medicine, Kuwait University, Kuwait
}

\section{Key Words}

Benign prostatic hyperplasia $\cdot$ Urinary retention • Transurethral microwave thermotherapy • Indwelling urethral catheter

\begin{abstract}
Objective: The aim of this preliminary study was to evaluate the efficacy of Prostalund feedback treatment (PLFT) using microwave thermotherapy equipment for benign prostate hyperplasia (BPH) in patients with urinary retention and an indwelling urethral catheter. Subjects and Methods: Four unselected patients, 51-68 years old (mean age 60 years), with urinary retention and an indwelling catheter were treated with PLFT under local anaesthesia and sedation. They were considered high-risk patients for treatment by transurethral resection of the prostate (TURP) under spinal or general anaesthesia. Initial assessment included prostate-specific antigen, determination of prostate volume and exclusion of prostate cancer using transrectal ultrasound (TRUS). The patients had the indwelling catheter for 1-2 weeks prior to treatment, the urethral catheters were removed 10-12 days after PLFT. Two patients who failed to void after transurethral microwave thermotherapy underwent urodynamic study to determine the reason for failure. Result: The mean volume of the treated prostate gland was
\end{abstract}

\section{KARGER}

Fax +4161306 1234 E-Mail karger@karger.ch www.karger.com
(C) 2005 S. Karger AG, Basel

$1011-7571 / 05 / 0144-0272 \$ 22.00 / 0$

Accessible online at:

www.karger.com/mpp
$75.50 \mathrm{~cm}^{3}$ (range $\left.51-150 \mathrm{~cm}^{3}\right)$. Two of the 4 patients were successfully relieved of their indwelling catheter with satisfactory peak flow, residual urine and symptom score, while treatment failed in the remaining 2 . The reasons for the failure were identified as a small bladder capacity and neurogenic bladder in a diabetic patient and the presence of a middle lobe with large protruding lateral lobes into the bladder of the other patient. The latter patient had a prostate volume of $150 \mathrm{~cm}^{3}$. 'Salvage' TURP in both patients revealed that PLFT caused extensive necrosis of prostatic tissue and the TURP was then accomplished with minimal blood loss $(<200 \mathrm{ml})$ even in the patient with 150-gram prostate. There were no serious complications such as bleeding, sepsis, rectoprostatic fistula or urine incontinence. Conclusion: The result of this preliminary investigation appears to be satisfactory and indicates that PLFT could be a good, minimally invasive alternative to surgery for $\mathrm{BPH}$ patients with retention of urine and an indwelling catheter.

Copyright (C) 2005 S. Karger AG, Basel

\section{Introduction}

Prostatic hyperplasia is a common urological disorder in ageing men. The incidence is rising all over the world due to increasing life expectancy in most societies. The 
gold standard for treating symptomatic benign prostatic hyperplasia $(\mathrm{BPH})$ remains transurethral resection of the prostate (TURP), usually performed under (local or general) anaesthesia [1]. Nearly $30 \%$ of men with symptomatic BPH who require TURP are elderly and often with significant co-morbid diseases of cardiovascular, respiratory, and renal systems. These patients may be denied surgical intervention on account of being poor anaesthetic risks and for them, it may mean spending the rest of their life carrying an indwelling urethral or suprapubic catheter. This method of palliation is not only unsatisfactory but a source of additional morbidity for these elderly patients. In the last 10 years several non-surgical, minimally invasive methods for ablating $\mathrm{BPH}$ tissue have been developed for use in an 'office setting' using local anaesthesia and sedation. Such methods of treatment include thermotherapy, cryogenic, chemical or enzymatic tissue ablation [1,2]. Since their introduction, the relevant equipment has been modified to make them safer, to reduce complication rate, and ensure that results obtained from their use are equal to those of TURP [3].

In this preliminary investigation, we assessed the use of a 'fourth-generation' transurethral microwave thermotherapy (TUMT) machine called 'Prostalund' (Prostalund, AB, Lund, Sweden) during a workshop from 10.10.03 to 14.10 .03 in Kuwait.

\section{Subjects and Methods}

Four unselected patients, 51-68 years old (mean age 60 years) with urinary retention and an indwelling catheter were treated with Prostalund Feedback Thermotherapy (PLFT). All the 4 patients had $\mathrm{BPH}$, considerable prostatism or lower urinary tract symptoms suggestive of BPH before presenting with acute urinary retention and were considered poor anaesthetic risks with American Society of Anaesthesiology (ASA) grade $>3$ for TURP. They signed an informed consent form. The mean urine volume drained by urethral catheters at presentation was $790 \mathrm{ml}$ (range 700-1,500). Pre-operative assessment included digital rectal examination, estimation of prostate-specific antigen (PSA) estimation of prostate volume using transrectal ultrasound and exclusion of prostate cancer. The patients had an indwelling catheter for 1-2 weeks prior to treatment. Treatment was carried out under lignocaine hydrochloride jelly (Instillagel, Farco-Pharma GmbH, Cologne, Germany) intraurethral instillation (local anaesthesia) and sedation using oral diazepam $(10 \mathrm{mg})$ given $2 \mathrm{~h}$ prior to start of treatment. During treatment using PLFT, if the patient was agitated or experiencing pain, low doses of intravenous pethidine (10-50 mg) and or diazepam (5-10 mg) were given. The urethral catheters were removed 10-12 days after PLFT. Two patients who were unable to void after catheter removal had a urodynamic study to find out the cause of failure of treatment. The patients then had cystoscopy and 'salvage' TURP after signing a high-risk consent form for the procedure. The extent of tissue necrosis caused by preliminary treatment with TUMT and the effect on blood loss at TURP were noted.

\section{Results}

The volume of the prostate in the 4 patients was 51 , 53,48 and $150 \mathrm{~cm}^{3}\left(\right.$ mean $\left.=75.50 \mathrm{~cm}^{3}\right)$. PSA was normal and transrectal ultrasound of the prostate revealed $\mathrm{BPH}$ with no evidence of prostate cancer. Following the removal of the indwelling catheter 10-14 days later, 2 of the patients voided urine satisfactorily with peak flow rate $>15 \mathrm{ml} / \mathrm{s}$ and residual urine $<30 \mathrm{ml}$. The remaining 2 patients ( 1 with prostate volume of $52 \mathrm{~cm}^{3}$ and the other $150 \mathrm{~cm}^{3}$ ) went into urinary retention and required further periods of urethral catheterization and reassessment including urodynamic study. Post-TUMT urodynamic study confirmed a small bladder capacity $(<200 \mathrm{ml})$ in the patient with prostate volume of $52 \mathrm{~cm}^{3}$, detrusor instability and poor bladder compliance. The patient was a known diabetic. Cystoscopy confirmed extensive prostate necrosis, but a very 'high bladder' neck that required incision and minimal TURP combined with anticholinergic treatment for bladder instability; the patient voided satisfactorily. The other patient with a 150 -gram prostate was subjected to 'salvage' TURP 6 weeks after the initial TUMT. At cystoscopy in the 2 patients, it was observed that the TUMT produced extensive necrosis of prostatic tissue. Histological examination of resected prostatic tissue confirmed extensive necrosis with little inflammatory response (fig. 1, 2). TURP was performed with minimal loss of blood $(<200 \mathrm{ml}$ in each patient) in these 2 patients.

The postoperative course of the 2 patients was uneventful. Nine months after treatment all 4 patients were voiding satisfactorily without any complications of TUMT or TURP.

\section{Discussion}

Our preliminary experience indicates that the PLFT is a useful, effective and minimally invasive technique for treating patients with $\mathrm{BPH}$. This method of treating $\mathrm{BPH}$ will substantially reduce bed occupancy and reduce the overall cost of treating patients with BPH as it can be carried out in an office setting under local anaesthesia and sedation [1]. The reasons for failure of the technique in our patients were: the presence of a significant middle 
Fig. 1. Histological appearance of prostatic tissues showing features of $\mathrm{BPH}$ without previous TUMT treatment. HE. $\times 320$.

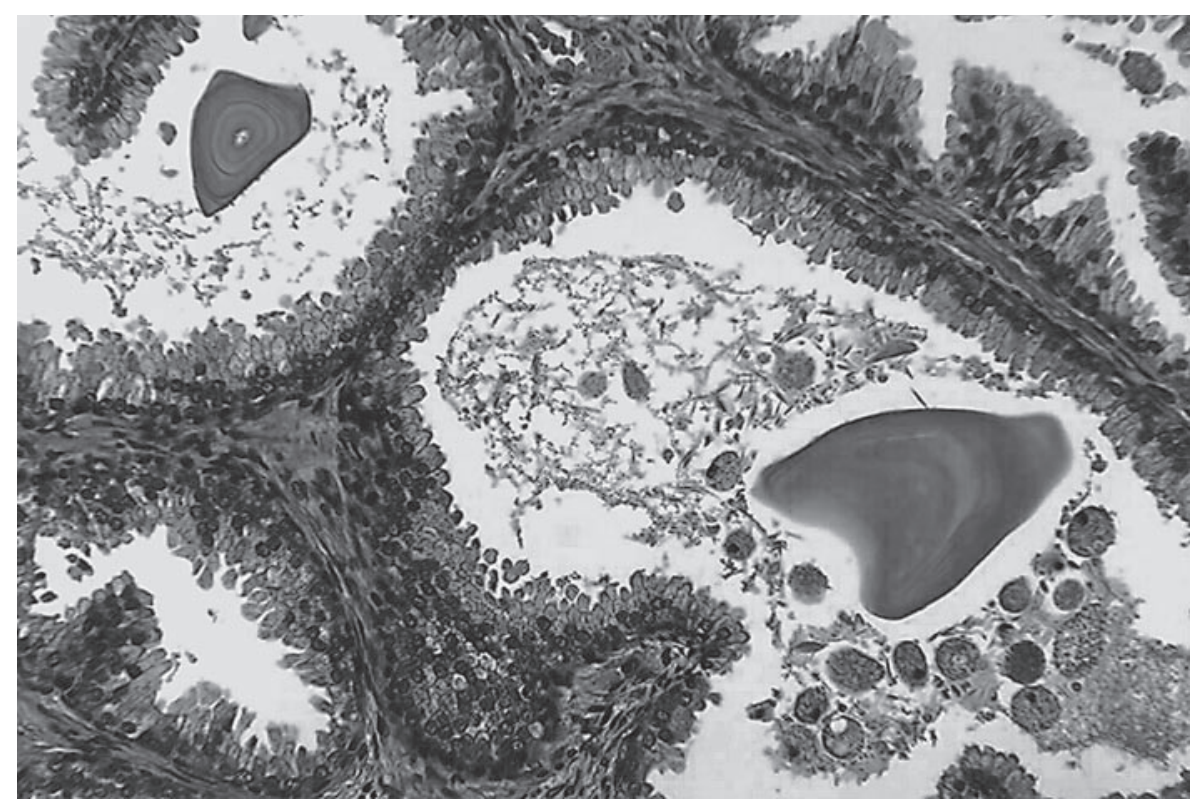

lobe prostatomegaly in the patient with the 150-gram prostate, coupled with lateral lobes that were very large with substantial amount of tissue protruding intravesically, that after TUMT prolapsed and formed a valve in the bladder neck. TURP was successfully performed in the patient with minimal blood loss and within $30 \mathrm{~min}$ as there were no bleeding vessels to deal with. Had a TURP been performed for such a patient ab initio because of the large prostate volume, the patient would have most likely required blood transfusion and would have required more than $1 \mathrm{~h}$ of resection - all of which would have resulted in higher morbidity or mortality. Another modality of treatment for such a patient would have been retropubic prostatectomy with all its known complications. Worse still, the patient could have been condemned to carrying a catheter for the rest of his life. The second patient where the technique failed had a small bladder capacity and urodynamic study confirmed features of diabetes-induced neurogenic bladder. These associated vesical anomalies secondary to diabetes mellitus may have contributed to the failure of treatment in this patient. Schelin [4], in a personal series of 19 patients who had Prostalund TUMT, also confirmed that patients with large lateral lobes and detrusor impairment do poorly after TUMT.

The use of medical treatment, e.g. $\alpha$-antagonists like alfuzosin, terazosin tamsulosin, in patients with acute urinary retention secondary to $\mathrm{BPH}$ is suitable for pa- tients with no previous history of prostatism or lower urinary tract symptoms suggestive of $\mathrm{BPH}$ and with prostate volume less than $40 \mathrm{~cm}^{3}$ [5-8]. The success rate of medical treatment is high in patients with such features or those where the underlying retention has been precipitated by temporary factors like anaesthesia, drugs or alpha-adrenergic sympathomimetic cold medications [6]. The patients used in this assessment of PLFT did not have these features, hence, they were not subjected to a preliminary period of trial without catheter with or without medical treatment since this modality of treatment was likely to be unsuccessful in these patients [7].

Currently, the postulated and known indications for TUMT include the following:

(a) Patients with symptomatic $\mathrm{BPH}$ who are not candidates for medical treatment especially if they are also considered very poor risk for anaesthesia.

(b) One-time treatment of patients with moderately enlarged prostate, i.e. prostate volume $>40$ and $<100 \mathrm{~cm}^{3}$.

(c) Initial treatment of patients with large prostates $(>100 \mathrm{~g})$, i.e. debulking the prostate and subsequent quick TURP, as demonstrated in 1 of our patients. This method of treating very large BPH will be akin to the wellknown urological principle of debulking treatment of staghorn renal calculus using percutaneous nephrolithotomy followed by lithotripsy to residual stone fragments. As illustrated by one of our patients, this method of treat- 


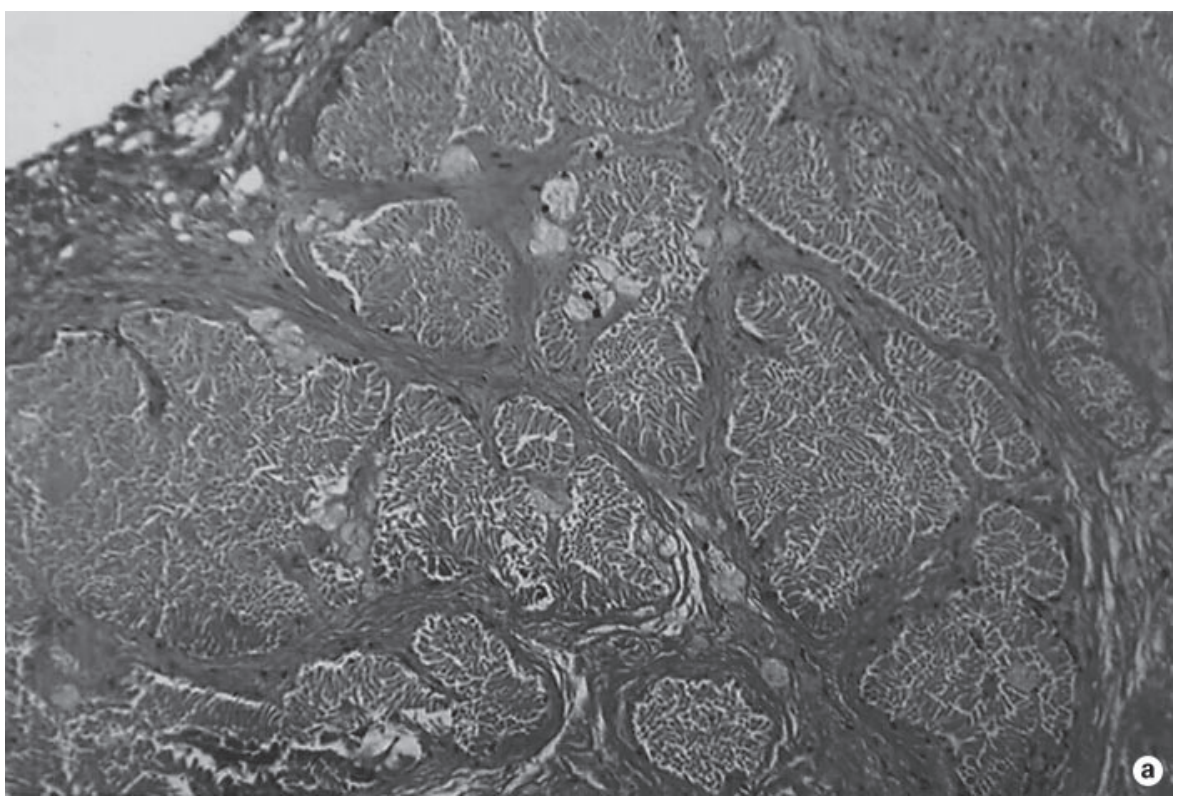

Fig. 2. a Histological appearance of prostatic tissue resected after TUMT showing extensive necrosis. HE. $\times 200$. b Histological appearance of prostatic tissue resected after TUMT showing extensive area of necrosis and minimal inflammatory response. HE. $\times 320$.

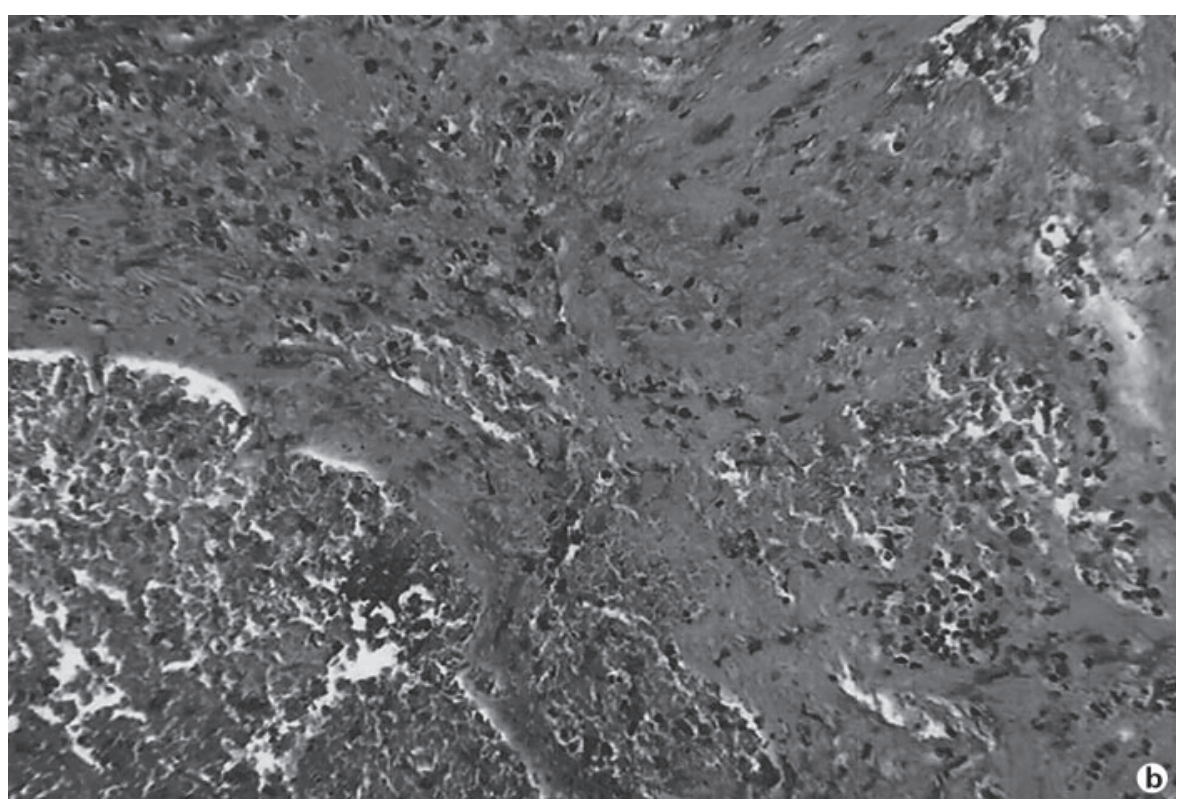

ment allows very large prostates to be treated without requiring blood transfusion or resorting to retropubic prostatectomy.

(d) It can be used to achieve 'channel TURP' in patients with locally advanced prostate cancer with concomitant bladder outlet obstruction.

(e) It can be used for treating patients with recalcitrant chronic prostatitis - when all else fails.

As the histological images (fig. 1,2) show extensive necrosis with little inflammatory reaction, this suggests that the method of 'kill' by microwaving is instantaneous and little inflammatory response is generated [2]. Thus, it would appear from our limited and preliminary experience that TUMT will destroy any focus of prostatic malignancy in its field of treatment (stage T0a or T1N0M0) - perhaps more effectively than TURP. Consequently, the lack of tissues for histological examination may not be of considerable hindrance to using the technique. Pretreatment digital rectal examination, PSA estimation and transrectal ultrasound examination of the 
prostate would have excluded any prostate cancer greater than stage $\mathrm{T} 1$.

This modality of treatment of patients with BPH has also been shown to be more cost-effective than TURP since it is possible for the treatment to be performed as an outpatient procedure [1,9]. Similarly, the retreatment rate has been shown in large series of patients followed for about 3 years to be about the same as that for TURP - the current gold standard with which all other modalities of treating BPH is to be compared $[1,5,9]$.

The PLFT thermotherapy machine used in this preliminary study is a fourth-generation thermotherapy equipment which has several advantages over other devices. The key advantage of the Prostalund TUMT over other devices in its category includes a specially designed catheter with an intraprostatic antenna and cooling systems for the rectum and urethra. These features ensure that the area of the prostate to be ablated is carefully monitored (similar to when performing TURP) and minimizes inadvertent injuries to the urethra or the rectum. Also, since the treatment is computer-controlled, and the equipment has thermosensors, the machine has been designed to 'shut down' automatically when the local temperature exceeds $45^{\circ} \mathrm{C}$. This is another important safety feature, designed to minimize complications. The 'Prostalund' machine can therefore be said to be the most advanced TUMT equipment currently available on the market because it has all the features that a standard
'TUMT' machine should have, namely 'controlled tissue ablation with built-in safety features' [2, 3]. Furthermore, the Prostalund TUMT can be carried out as an outpatient procedure under local anaesthesia and sedation, thus making it possible to treat virtually all patients with symptomatic BPH irrespective of the presence of other co-morbid disease.

\section{Conclusion}

This preliminary assessment indicates that PLFT is a minimally invasive technique of treating $\mathrm{BPH}$ that could be a realistic alternative in the management of patients with symptomatic BPH. We recommend this modality of treating patients with BPH for further 'clinical trials' in various parts of the world.

\section{Acknowledgements}

We would like to thank Mrs. Rada Lempert of Prostalund AB, Lund, Sweden and their local sponsors in Kuwait, Al-Essa Medical $\&$ Scientific Equipment Co. W.L.L., Kuwait, for allowing us to use the PLFT equipment free of charge during the workshop. We wish to thank Prof. J.T. Anim of the Department of Pathology, Kuwait University for providing detailed histological examination of surgical specimens.

\section{References}

1 Wilt TJ: Treatment options for benign prostatic hyperplasia. BMJ 2002;324:1047-1048.

-2 Larson BT, Bostwick DG, Conica AG, Larson TR: Histological changes of minimally invasive procedures for the treatment of benign prostatic hyperplasia and prostate cancer: Clinical implications. J Urol 2003;170:12_ 19.

-3 Wagnell L, Schelin S, Nardling J, Richthoff J, Magnasson B, Schain M, et al: Feedback microwave thermotherapy versus TURP for clinical BPH: A randomized controlled multicenter study. Urology 2002;60:292-299.

-4 Schelin S: Microwave thermotherapy in patients with benign prostatic hyperplasia and chronic urinary retention. Eur Urol 2001;39: 400-404.
-5 Roehrborn C: AUA guideline on management of benign prostatic hyperplasia (2003). Chapter 1: Diagnosis and treatment recommendations AUA Practice Guidelines Committee. J Urol 2003; 170:530-547.

6 6 De Mey C, Michel MC, McEwen J, Moreland $\mathrm{T}$ : A double-blind comparison of terazosin and tamsulosin on their differential effects on ambulatory pressure and nocturnal orthostatic stress testing. Eur Urol 1998;33:481-487.
7 Chung BH, Ko WJ, Kim SI, Hong SJ: Identification of baseline clinical factors that predict maintenance for medical treatment of $\mathrm{BPH}$. Eur Urol 2002;1(suppl 1):106.

8 de la Rosette JJ, Kortmann BB, Rossi C, Sonke GS, Floratos DL, Kiemeney LA: Long-term risk of re-treatment of patients using alphablockers for lower urinary tract symptoms. J Urol 2002;167:1734-1739.

-9 De La Rosette JJMCH, Floratos DL, Severens JL, Kiemeney LA, Debruyne FMJ, Pilar-Laguna M: Transurethral resection versus microwave thermotherapy of the prostate: A costconsequences analysis. BJU Int 2003;92: 713-718. 